\title{
Expression of p27(Kip1), a Cyclin-Dependent Kinase Inhibitor, in Human Peripheral Blood Mononuclear Cells Is Inversely Associated with Potential Carcinogenic Risk in Obese Type 2 Diabetic Individuals Relative to Lean Normal Controls
}

\author{
Isao Eto \\ Department of Nutrition Sciences, University of Alabama at Birmingham, Birmingham, USA \\ Email: etoi@uab.edu
}

Received 11 April 2014; revised 10 May 2014; accepted 9 June 2014

Copyright (C) 2014 by author and Scientific Research Publishing Inc.

This work is licensed under the Creative Commons Attribution International License (CC BY). http://creativecommons.org/licenses/by/4.0/

(c) (i) Open Access

\begin{abstract}
Introduction: The consensus report issued jointly by the American Diabetes Association and the American Cancer Society stated that "type 2 diabetes and cancer share many risk factors, but potential biologic links between the two diseases are incompletely understood". Interestingly, however, a recent report suggested that the expression of p27(Kip1), a cell cycle repressor protein, in the rodent liver was inversely associated with potential carcinogenic risk in the genetic rodent models of diabetic obesity. p27 is a cyclin-dependent kinase inhibitor that, when down-regulated, allows the progression of the cell cycle from G1 to $S$ phase, thereby increasing the risk of developing cancer. Objective: The objective of the study described below was to extend the results of the recent report on the expression of p27 in the livers of obese, diabetic rodents to the humans and investigate whether the expression of p27 in the human peripheral blood mononuclear cells (PBMCs) might also be inversely associated with potential carcinogenic risk in obese type 2 diabetic individuals relative to the lean normal controls. Methods: Western immunoblot analysis was performed to evaluate the expression of p27 and the two most relevant upstream molecular signaling pathways of the expression of p27, namely 4E-BP1 and MNK1, in human PBMCs obtained from obese type 2 diabetic individuals relative to the lean normal controls. Results: First, expression of p27 in human PBMCs was significantly down-regulated in obese type 2 diabetic indivi-

How to cite this paper: Eto, I. (2014) Expression of p27(Kip1), a Cyclin-Dependent Kinase Inhibitor, in Human Peripheral Blood Mononuclear Cells Is Inversely Associated with Potential Carcinogenic Risk in Obese Type 2 Diabetic Individuals Relative to Lean Normal Controls. American Journal of Molecular Biology, 4, 114-128.
\end{abstract}

http://dx.doi.org/10.4236/ajmb.2014.43013 
duals relative to the lean normal controls. Secondly, expression of p27 in human PBMCs was also significantly down-regulated in obese type 2 diabetic African Americans relative even to the obese type 2 diabetic Caucasian Americans. Conclusions: Expression of p27 in human PBMCs was inversely associated with potential carcinogenic risk in obese type 2 diabetes relative to the lean normal controls.

\title{
Keywords
}

\author{
Zucker Rats, Liver, Kidney, Diabetic Nephropathy, 4E-BP1, MNK1, eIF4E
}

\section{Introduction}

The consensus report issued jointly by the American Diabetes Association and the American Cancer Society stated that "type 2 diabetes and cancer share many risk factors, but potential biologic links between the two diseases are incompletely understood [1]". Interestingly, however, a recent report suggested that the expression of p27(Kip1), a cell cycle repressor protein, in the rodent liver was inversely associated with potential carcinogenic risk in the genetic rodent models of diabetic obesity [2].

p27(Kip1) is a cyclin-dependent kinase inhibitor that, when down-regulated, allows the progression of the cell cycle from G1 to S phase, thereby increasing the rate of DNA replication in the S phase and the risk of developing cancer. Conversely, when up-regulated, p27 primarily inhibits the progression of the cell cycle from G1 to S phase, thereby decreasing the rate of DNA replication in the S phase and the risk of developing cancer.

Previously, it was reported that the female obese diabetic Zucker rats exhibited increased incidence of 7,12-dimethylbenz(a)anthracene (DMBA)-induced rat mammary adenocarcinomas compared with female lean normal Zucker rats [3]-[9]. And a recent report suggested that the hepatic expression of p27 was significantly down-regulated — by about 20\% - in obese diabetic Zucker rats relative to the lean normal Zucker rats [2]. Previously, it was also reported that the homozygous long-lived Ames dwarf mice had a delayed occurrence of presumably fatal neoplastic disease compared with their heterozygous normal siblings [10] and that the incidence of presumably fatal adenocarcinomas in the lung was significantly lower in homozygous Ames dwarf mice relative to their heterozygous normal siblings [10] [11]. The investigators of these previous studies noted that the homozygous long-lived Ames dwarf mice had physiological characteristics that were similar to the effects of dietary restriction [10]. Again, a recent report suggested that the hepatic expression of p27 was significantly up-regulated — by about 60\% - in homozygous long-lived Ames dwarf mice relative to the heterozygous normal control Ames mice [2]. As for the study of the expression of p27 in human breast cancer cells in vitro, various nutritional or chemopreventive anti-cancer agents, including retinoic acids, 4-hydroxitamoxifen, resveratrol, curcumin, and others, significantly increased the expression of p27 in these cells and, conversely, various nutritional pro-cancer agents, including glucose, insulin and branched-chain amino acids, significantly decreased the expression of p27 in these cells [12]-[14].

The effects of various pro- and anti-cancer agents on the expression of p27 were unique to p27, not seen with any other G1-to-S phase cell cycle regulatory proteins including p21 Cip1/Waf1, another cyclin-dependent kinase inhibitor. The molecular mechanism of the expression of p27 is also unusual. Its expression is regulated primarily at the translational [13] [15] [16] and post-translational levels, rather than at the transcriptional level.

The results presented in the recent report above have now been extended to the human peripheral blood mononuclear cells (PBMCs). The objective of the new study described below was to investigate whether the expression of p27 in the human PBMCs might also be inversely associated with potential carcinogenic risk in obese type 2 diabetic individuals relative to the lean normal controls.

There was, however, a slight concern in achieving this objective because the expression of p27 had been well-known to be directly, rather than inversely, associated with the severity of the diabetic nephropathy in obese type 2 diabetes. For example, the lack of p27 was reported to ameliorate progression of diabetic nephropathy in diabetic p27 knockout (p27 -/-) mice relative to diabetic p27 wild-type (p27 +/+) mice [17] [18]. Conversely, glomerular p27 protein — but not p27 mRNA—expression was strongly enhanced in diabetic $d b / d b$ mice compared with non-diabetic $d b /+$ littermates [19] [20]. Immunohistochemical studies revealed that this stimulated expression of p27 was mainly restricted to the nuclei of mesangial cells and podocytes, but glomeru- 
lar endothelial cells occasionally also were stained positively [19].

Thus, it was quite possible that the expression of p27 in PBMCs could be directly, rather than inversely associated with the risk of developing cancer in obese type 2 diabetes. However, the results suggested otherwise. The expression of p27 in PBMCs was significantly down-regulated, rather than up-regulated in the human PBMCs from obese type 2 diabetic individuals relative to the lean normal controls. Additionally, the results further suggested that the expression of p27 was also significantly down-regulated, rather than up-regulated in the human PBMCs from obese type 2 diabetic African Americans relative even to the obese type 2 diabetic Caucasian Americans.

\section{Materials and Methods}

\subsection{Cryopreserved Human Peripheral Blood Mononuclear Cells (PBMCs)}

Cryopreserved human peripheral blood mononuclear cells (PBMCs) from obese type 2 diabetic individuals and lean normal controls were purchased from ZenBio, Inc. (Research Triangle Park, North Carolina, USA). All donors of PBMCs were tested at the ZenBio, Inc. for blood-borne pathogens (HIV-1, HIV-2, HTLV I, HTLV II, syphilis, CMV, and hepatitis C). In addition, the cell population distribution of PBMCs was characterized at the ZenBio, Inc. using fluorescence flow cytometry. To comply with US Food and Drug Administration (FDA) regulations, the use of these cells were restricted for research only and not approved for human or veterinary or in diagnostic or clinical procedures. Since PBMCs are the primary cells of human origin, they were treated as potentially contaminated biological specimens even if accompanying serological reports were negative and they were handled by establishing or following appropriate safety control procedures (Biosafety Level 2 or higher) to ensure the safety of using these cells. For example, the gloves and masks were always worn and work was performed behind a protective screen. Also, all media, supplements, and cell culture ware used were sterile.

Each vial of the cryopreserved PBMCs was provided with the following information about the donor of the PBMCs: age (overall range 26 to 68 years old; overall average 39.1 years old; range of the lean normal controls 26 to 41 years old; and obese type 2 diabetic individuals 32 to 68 years old; average of lean normal controls 31.3 years old; and obese type 2 diabetic individuals 42.1 years old), gender (overall average number of males $57.1 \%$ and females $42.9 \%$ ), pathogen (100\% negative/none positive), race (overall average number of African Americans 20.8\% and Caucasian Americans 79.2\%; average number of lean normal African Americans 27.3\% and Caucasian Americans 72.7\% and average number of obese type 2 diabetic African Americans 15.4\% and Caucasian Americans 84.6\%), BMI (body mass index), Hgb (hemoglobin) A1c\%, cell population distributions (CD3+ (T cells), CD4+ (Th cells), CD8+ (Tc cells), CD19+ (B cells), and CD56+ (NK cells)) and propidium iodide \% viability. The donors were fasted at the time of the collection of the blood. Each vial of cryopreserved PBMCs was labeled as obtained from either lean normal or obese type 2 diabetic donor(s).

Upon shipment receipt, vials of the cryopreserved PBMCs were immediately stored in liquid or vapor phase nitrogen. Each vial of the cryopreserved human PBMCs contained a minimum of $15.0 \times 10^{6}$ cells for unfractionated cells and a minimum of $3.0 \times 10^{6}$ cells for fractionated cells.

\subsection{Preparation of Soluble Protein Extracts from Cryopreserved Human Peripheral Blood Mononuclear Cells (PBMCs)}

Twenty five (25) ml of unsupplemented Dulbecco's Modified Eagle’s Medium (DMEM) (Sigma-Aldrich, Inc., St. Louis, Missouri, USA) were warmed to $37^{\circ} \mathrm{C}$. The vial of cryopreserved human PBMCs (peripheral blood mononuclear cells) was then rapidly thawed in a $37^{\circ} \mathrm{C}$ water bath until just prior to complete thawing (slurry of residual ice should be present). The cell suspension was aseptically transferred to a $50 \mathrm{ml}$ conical tube. The vial was rinsed with $1 \mathrm{ml}$ of unsupplemented DMEM and then the remainder of the warmed unsupplemented DMEM was slowly added drop wise into the $50 \mathrm{ml}$ conical tube until the total volume reached $25 \mathrm{ml}$. The cell suspension was then centrifuged at $200 \times \mathrm{g}$ at room temperature for 15 minutes. The supernatant was carefully removed and the precipitated cells were gently resuspended to 75 to $200 \mu \mathrm{l}$ of the $+4^{\circ} \mathrm{C}$ RIPA Lysis Buffer (Santa Cruz Biotechnology, Dallas, Texas, USA) supplemented with sodium fluoride (Sigma-Aldrich, Inc.), phenylmethylsulfonyl fluoride(PMSF) (Santa Cruz Biotechnology), protease inhibitor cocktail (Santa Cruz Biotechnology) and sodium orthovanadate (Santa Cruz Biotechnology).

The cells resuspended in the $+4^{\circ} \mathrm{C}$ RIPA Lysis Buffer were placed in an ice bucket and sonicated three times for 4 to 5 seconds each using Sonic Dismembrator Model 100 (Fisher Scientific USA, Pittsburgh, Pennsylvania, 
USA). The soluble protein extract was then collected by centrifugation at $10,000 \times \mathrm{g}$ for 30 seconds at $+4^{\circ} \mathrm{C}$ and the aliquots of the extract were stored at $-80^{\circ} \mathrm{C}$ in an ultra-low freezer.

\subsection{Western Immunoblot Analysis of the Soluble Protein Extracts from Cryopreserved Human Peripheral Blood Mononuclear Cells (PBMCs)}

The following primary antibodies were purchased from the Cell Signaling Technology (Danvers, Massachusetts, USA): 1) 4E-BP1 (total) and phospho-4E-BP1 (Thr37/46); 2) phospho-MNK1 (Thr197/202); 3) eIF4E (total) and phospho-eIF4E (Ser209); and 4) GAPDH; Additionally, the following primary antibodies were obtained from the Santa Cruz Biotechnology (Dallas, Texas, USA): 1) p27Kip1; 2) 4E-BP1; 3) MNK1; 4) eIF4E; and 5) GAPDH.

For western immunoblot analysis, the soluble protein extracts (10 to $50 \mu$ g protein per lane) were applied to the SDS-PAGE and, after fractionation, transferred to the nitrocellulose membrane, which was then blocked and incubated in a solution containing first primary antibody. After shaking overnight at $+4^{\circ} \mathrm{C}$, the target proteins bound to the first primary antibody were further incubated in a solution containing alkaline phosphatase (AP)-conjugated secondary anti-immunoglobulin antibody and detected by chemiluminescence using TROPIX Western-Star Kit (Applied Biosystems, Foster City, California, USA). After exposure to X-ray film, the blots were stripped using Western Re-Probe Solution (G-Biosciences, St. Louis, Missouri, USA), checked for removal of the chemiluminescence and then re-probed with second primary antibody.

Densitometric measurements of the intensity of the bands on the X-ray films were performed using UN-SCAN-IT Gel \& Graph Digitizing Software Version 6.1 (Silk Scientific, Orem, Utah, USA). Background corrections were done by four corner interpolation method and optical density calculations were performed using linear standard reflective scan method.

\subsection{Statistical Analysis}

An experimental value with statistical significance of $\mathrm{P} \leq 0.05$ compared to the control by $\mathrm{t}$ test is indicated as a single asterisk on top of the vertical bar.

\section{Results}

Commercially available cryopreserved human PBMCs were used to investigate the expression of p27(Kip1) in human peripheral blood mononuclear cells (PBMCs) from obese type 2 diabetic and lean normal donors, Purchasing these cells did not require approval from the Institutional Review Board (IRB)(University of Alabama at Birmingham. Birmingham, Alabama, USA). Each vial of the cryopreserved cells was labeled as obtained from either obese type 2 diabetic individuals or lean normal donors. Additionally, each vial was provided with various informations, including age, gender, race, and cell population distribution, about the donor of the PBMCs as described in the Materials and Methods section.

\subsection{Expression of p27(Kip1) Was Significantly and, in Certain Cases, Severely Decreased in Human Peripheral Blood Mononuclear Cells (PBMCs) Obtained from Obese Type 2 Diabetic Donors Relative to Lean Normal Donors}

Western immunoblot analysis of the expression of p27 in human peripheral blood mononuclear cells (PBMCs) -Panels a to d of Figure 1 shows that, without exception, the expression of p27 was significantly decreased in human PBMC samples obtained from obese type 2 diabetic donors relative to the lean normal donors. These results suggested that the risk of developing obesity-related cancer might be significantly decreased in lean normal individuals (see the diagram in the Panel a of Figure 2) or, conversely, the risk of developing obesity-related cancer might be significantly increased in obese type 2 diabetic individuals (Panel b of Figure 2).

Although expression of p27 in human PBMCs was significantly decreased in obese type 2 diabetic individuals relative to the lean normal individuals, extent of the decrease was quite variable; it ranged from $20 \%$ to over 95\%. In obese type 2 diabetic African Americans (AAs), expression of p27 in PBMCs was most severely depressed-by over 95\% - relative to the lean normal African Americans (AAs). By contrast, in obese type 2 diabetic Caucasian Americans (CAs), extent of the decrease in the expression of p27 in PBMCs was quite variable -by 20\% to 86\% - relative to the lean normal Caucasian Americans (CAs). None of the factors of donors other 


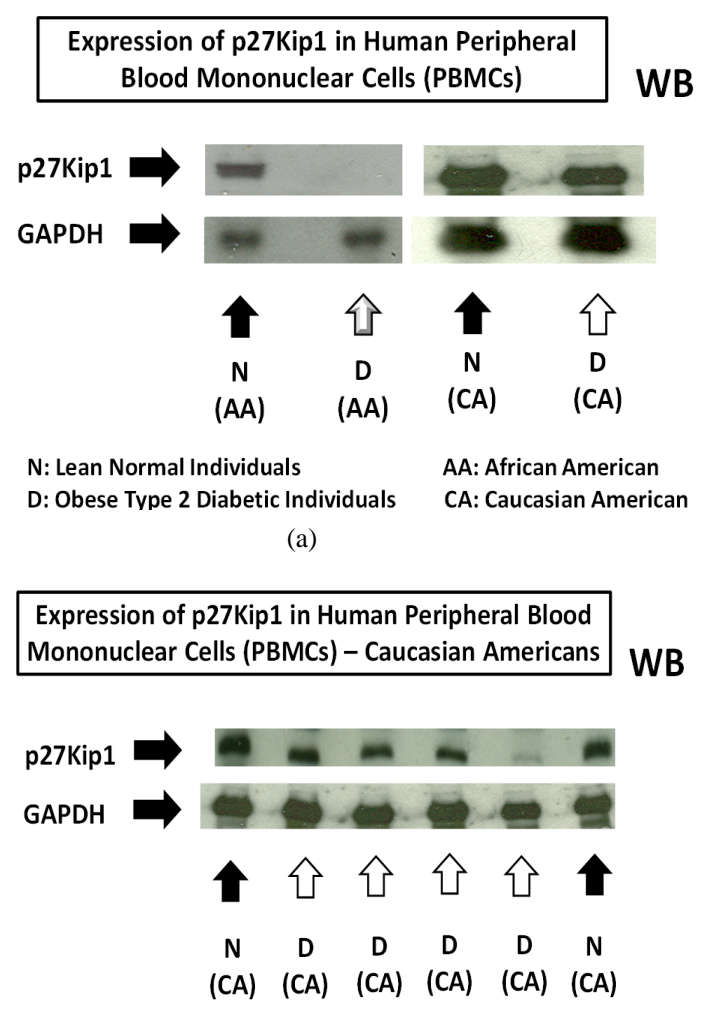

$\mathrm{N}$ : Lean Normal Individuals D: Obese Type 2 Diabetic Individuals

CA: Caucasian American

(c)
Expression of p27Kip1 in Human Peripheral Blood

Mononuclear Cells (PBMCs) - African Americans WB

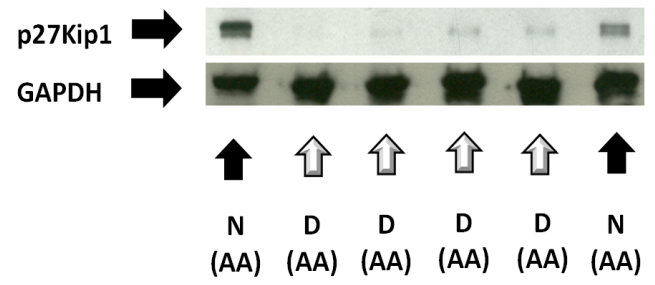

$\mathrm{N}$ : Lean Normal Individuals AA: African American D: Obese Type 2 Diabetic Individuals

(b)

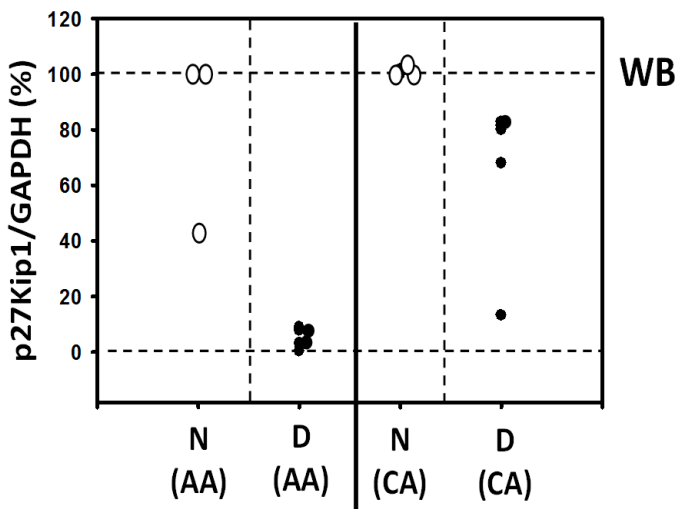

(d)

Figure 1. Expression of p27(Kip1) is significantly and, in certain cases, severely decreased in human peripheral blood mononuclear cells (PBMCs) obtained from obese type 2 diabetic individuals relative to the lean normal individuals. Panels a to $d$ show the results of the western immunoblot analysis of the expression of p27 in human PBMCs obtained from (Panel a) lean normal African American (AA) (n=1), obese type 2 diabetic African American (AA) $(n=1)$, lean normal Caucasian American (CA) $(n=1)$ and obese type 2 diabetic Caucasian American (CA) ( $=1$ ), (Panel b) lean normal African Americans (AAs) $(n=2)$ and obese type 2 diabetic African Americans (AAs) $(n=4)$, (Panel c) lean normal Caucasian Americans (CAs) $(n=2)$ and obese type 2 diabetic Caucasian Americans (CAs) $(n=4)$, and (Panel d) summary graph of the expression of $\mathrm{p} 27$ in human PBMCs presented in the Panels a, b and c.

than the race could account for the severe depression of the expression of p27 in PBMCs from obese type 2 diabetic African Americans. These observations need to be verified, however, using larger number of PBMC samples, but the number of commercially available PBMC samples was limited.

Another important issue that was needed to be investigated was the molecular mechanisms of how the upstream molecular signaling pathways of the expression of p27 was so severely depressed—by over $95 \%$-in the PBMCs obtained from obese type 2 diabetic African Americans relative to the lean normal African Americans and, for that matter, relative even to the obese type 2 diabetic Caucasian Americans. This issue will be addressed next. It would be helpful to note, in this context, that the expression of p27 in the livers of homozygous, obese diabetic rats or mice decreased by only $20 \%$, not by over $95 \%$, relative to the heterozygous, lean normal controls [2].

\subsection{Cell Population Distributions of Human PBMCs in Obese Type 2 Diabetic Donors Were Not Significantly Different from Those in Lean Normal Donors. Likewise, the Cell Population Distributions of PBMCs in Obese Type 2 Diabetic African Americans (AAs) Were Not Significantly Different from Those in Obese Type 2 Diabetic Caucasian Americans (CAs)}

To initiate the investigation of the upstream molecular signaling pathways of the expression of p27 in PBMCs, 
Decreased Risk of Developing Cancer

G1 to S Phase Cell Cycle Regulatory Proteins

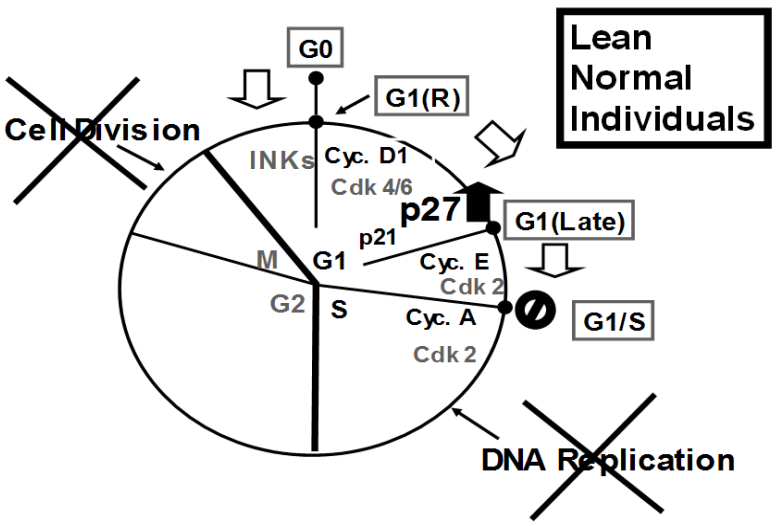

(a)

Increased Risk of Developing Cancer

G1 and S-Phase Cell Cycle Regulatory Proteins

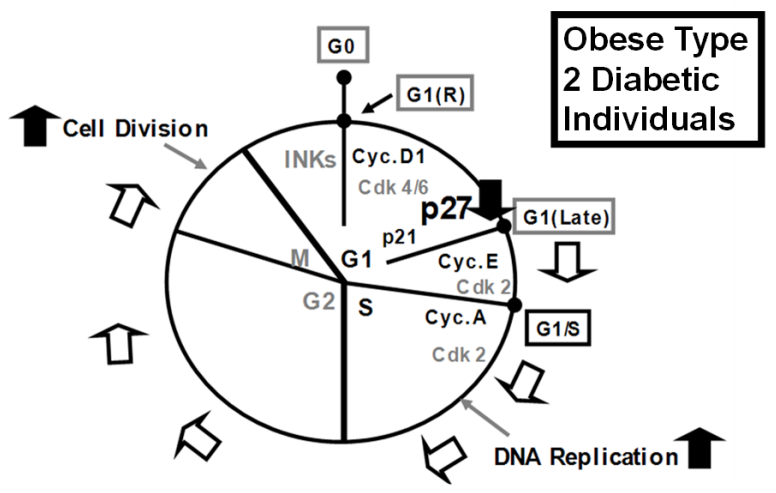

(b)

Figure 2. Regarding the results presented in Figure 1, panels a and e present hypothetical diagrams of (Panel a) how the higher levels of the expression of p27 could decrease the risk of developing cancer in lean normal individuals and (Panel e) how the lower levels of the expression of p27 could increase, rather than decrease, the risk of developing cancer in obese type 2 diabetic individuals.

the cell population distributions of PBMCs were compared between obese type 2 diabetic donors and lean normal donors (Table 1). The objective of this study was to investigate whether there were any significant differences in the cell population distributions of PBMCs between the two types of donors that could potentially be associated with the decrease in the expression of p27 in the PBMCs from obese type 2 diabetic donors relative to the lean normal donors. The results presented in Table 1 did not suggest, however, that there were any significant differences in the cell population distributions of PBMCs between the two types of the donors. Likewise, there were no significant differences in the cell population distributions of PBMCs between obese type 2 diabetic African and Caucasian Americans (AAs and CAs) (Table 1).

It might be useful to note here that there may be subtle differences in the cell population distributions of PBMCs that could potentially be associated with the decrease in the expression of p27 in PBMCs from obese type 2 diabetic donors. For example, Th(CD4+) cells consist of three different sub-populations, i.e., virgin Th0 cells and two differentiated Th1 and Th2 cells, and the changes in any one or more of these three sub-populations might potentially be associated with the decreased expression of p27 in PBMCs from obese type 2 diabetic donors. 
Table 1. Cell population distributions of human peripheral blood mononuclear cells (PBMCs).

\begin{tabular}{ccccccc}
\hline & \multicolumn{3}{c}{ Lean Normal } & \multicolumn{3}{c}{ Obese Type 2 Diabetic } \\
\cline { 2 - 7 } CD Antigen & $\begin{array}{c}\text { \%Positive } \\
\text { (CA) }\end{array}$ & $\begin{array}{c}\text { \%Positive } \\
\text { (CA) }\end{array}$ & $\begin{array}{c}\text { \%Positive } \\
\text { (AA) }\end{array}$ & $\begin{array}{c}\text { \%Positive } \\
\text { (AA) }\end{array}$ & $\begin{array}{c}\text { \%Positive } \\
\text { (AA) }\end{array}$ & $\begin{array}{c}\text { \%Positive } \\
\text { (CA) }\end{array}$ \\
\hline CD3+ (T cells) & 48.7 & 66.7 & 64.6 & 61.3 & 68.9 & 60.0 \\
CD4+ (Th cells) & 40.9 & 43.2 & 43.8 & 21.8 & 49.0 & 48.9 \\
CD8+ (Tc cells) & 13.8 & 19.8 & 38.2 & 11.2 & 17.7 & 24.8 \\
CD14+ (Monocytes) & 25.4 & 13.3 & 21.8 & 15.1 & 20.5 & 22.4 \\
CD19+ (B cells) & 12.2 & 5.1 & 2.8 & 5.1 & 7.7 & 9.6 \\
CD56+ (NK cells) & 13.4 & 11.8 & 12.8 & 12.4 & 8.9 & 14.9 \\
CD4 +/CD8 + Ratio & 3.0 & 2.2 & 1.2 & 2.0 & 2.7 & 2.0 \\
T cells/Monocytes & 74.1 & 80.0 & 86.4 & 76.4 & 89.4 & 82.4 \\
T cells/Monocytes/B cells & 86.3 & 93.3 & 89.2 & 81.5 & 97.1 & 92.0 \\
T cells/Monocytes/B cells/NK cells & 99.7 & 105.1 & 102.0 & 93.9 & 106.0 & 106.9 \\
\hline
\end{tabular}

AA: African American; CA: Caucasian American; CD: Antigens assayed by fluorescence flow cytometry.

\subsection{Fractionation of PBMCs from Lean Normal Donors Suggested That All \\ Sub-Populations of PBMCs, Including T (CD3+) Cells and Monocytes (CD14+), Probably Expressed Approximately the Same Level of p27 as Unfractionated PBMCs}

Panels a and b of Figure 3 show that the levels of expression of p27 in the unfractionated PBMCs from lean normal African Americans (AAs) were not significantly different from those of lean normal Caucasian Americans (CAs).

Additionally, the Panels a, c and d of Figure 3 also present the results of the western immunoblot analysis of 4E-BP1 and MNK1. These results indicated that the 4E-BP1 and MNK1, the components of the two most relevant upstream molecular signaling pathways of the expression of p27, were expressed in the unfractionated PBMCs obtained from lean normal African Americans (AAs). They also indicated that the levels of expression of 4E-BP1 and MNK1 in the lean normal African Americans (AAs) were not significantly different from those of the lean normal Caucasian Americans (CAs). The diagrams in the Panels a and b of Figure 4 summarize the role of 4E-BP1 and MNK1 in the upstream molecular signaling pathways of the expression of p27 previously identified in 1) the livers of genetically homozygous obese diabetic rats and mice and heterozygous lean normal controls [2] and 2) the various human breast cancer cell lines in vitro [12]-[14].

The levels of expression of p27 in the sub-populations of PBMCs were investigated next. Generally, the PBMCs consist primarily of the following four sub-populations, namely T $(\mathrm{CD} 3+)$ cells $(48.7 \%-68.9 \%$ positive), monocytes (CD14+) (13.3\% - 25.4\% positive), natural killer (CD56+) cells ( $8.9 \%-14.9 \%$ positive), and B (CD19+) cells (5.1\% - 12.2\% positive). Since the sum of T (CD3+) cells and monocytes (CD14+) makes up the majority (62.0\% - 94.3\%) of the sub-populations of PBMCs, the levels of expression of p27 in T (CD3+) cells and monocytes $(\mathrm{CD} 14+)$ were investigated. The purities of the preparations of $\mathrm{T}(\mathrm{CD} 3+)$ cells and monocytes (CD14+) were approximately $97.6 \%$ and $93.0 \%$ positive, respectively. As shown in the Panels a and b of Figure 3, the levels of expression of p27 in either T (CD3+) cells or monocytes (CD14+) were not significantly different from those in the unfractionated PBMCs in either lean normal African or Caucasian Americans (AAs or CAs). Based on simple arithmetic calculations, it was likely that the natural killer (CD56+) cells and B (CD19+) cells from lean normal African or Caucasian Americans (AAs or CAs) also expressed comparable levels of p27 as those in unfractionated PBMCs. Results of the western immunoblot analysis presented in the Panels a, c and d of Figure 3 indicated that 4E-BP1 and MNK1 were also expressed in T (CD3+) cells and monocytes (CD14+) and the levels of expression of 4E-BP1 in these cells were comparable to those of unfractionated PBMCs. The levels of expression of MNK1 in the monocytes (CD+14) were also comparable to those of unfractionated PBMCs. However, the levels of expression of MNK1 in the T $(\mathrm{CD} 3+)$ cells were significantly 


p27Kip1
4E-BP1 (Total)
MNK1 (Total)
GAPDH
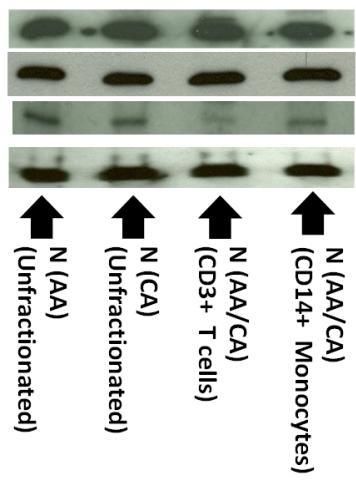

N: Lean Normal Individuals CA: Caucasian American AA: African American

(a)

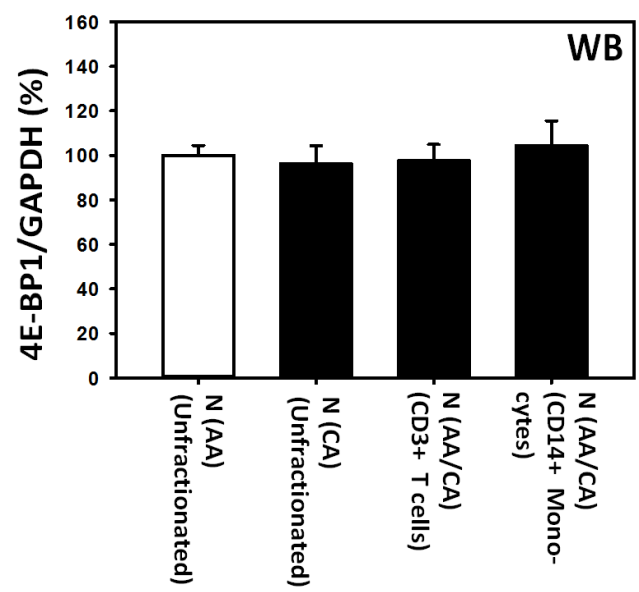

(c)

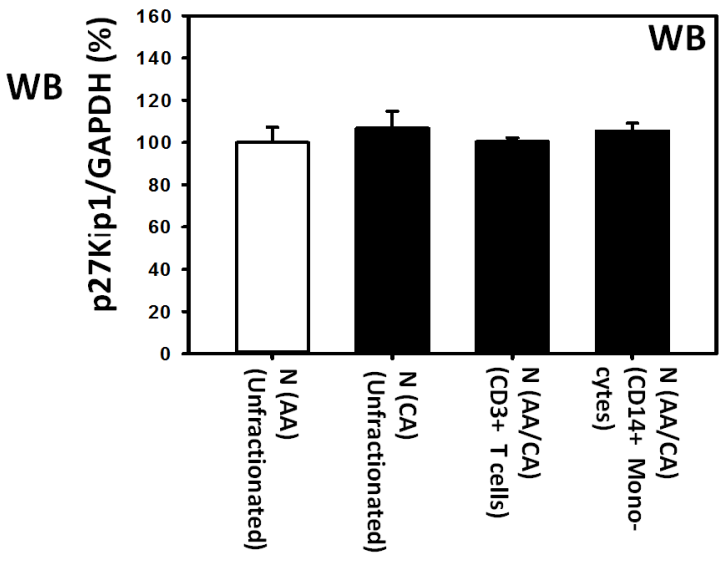

(b)

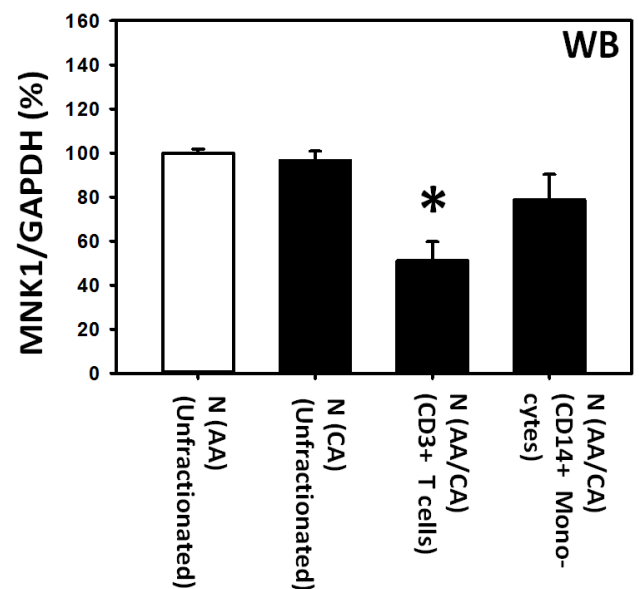

(d)

Figure 3. Fractionation of human peripheral blood mononuclear cells (PBMCs) from lean normal donors suggested that all sub-populations of PBMCs, including T (CD3+) cells and monocytes (CD14+), probably expressed approximately the same level of p27 as unfractionated PBMCs. Panels a to d show the results of the western immunoblot analysis of p27, total 4E-BP1 and total MNK1 in unfractionated PBMCs obtained from lean normal African Americans (AAs) $(n=3)$ and lean normal Caucasian Americans $(C A s)(n=3)$. Panels a to $\mathrm{d}$ also include the results of the western immunoblot analysis of p27, total 4E-BP1 and total MNK1 in T $(\mathrm{CD} 3+)$ cells $(\mathrm{n}=3)$ and monocytes $(\mathrm{CD} 14+)(\mathrm{n}=3)$ isolated from unfractionated PBMCs using Miltenyi's AutoMAC Simmunomagnetic cell sorting protocols (ZenBio Inc., Research Triangle Park, North Carolina, USA and Miltenyi Biotech Inc., Auburn, California, USA). The unfractionated PBMCs used to isolate $\mathrm{T}(\mathrm{CD} 3+)$ cells and monocytes (CD14+) were obtained from the mixtures of lean normal African and Caucasian Americans (AAs and CAs).

decreased — by about 40\% — relative to the unfractionated PBMCs. Surprisingly, this extent of the decrease in the expression of MNK1 in the $\mathrm{T}(\mathrm{CD} 3+)$ cells did not interfere with the expression of the p27 itself in the same $\mathrm{T}(\mathrm{CD} 3+)$ cells.

In summary, the results presented above suggested that all sub-populations of PBMCs, including T (CD3+) cells and monocytes (CD14+), probably expressed the same level of p27 as unfractionated PBMCs in either lean normal African or Caucasian Americans.

\subsection{Severe Depression-By over $95 \%$ - Of the Expression of p27 in the Peripheral Blood Mononuclear Cells (PBMCs) from Obese Type 2 Diabetic African Americans (AAs) Was Caused Primarily by the Severe Depression of MNK1 Upstream Molecular Signaling Pathways of the Expression of p27}

Last but certainly not least, the molecular basis of the cause of the severe depression—by over $95 \%$ —o the ex- 


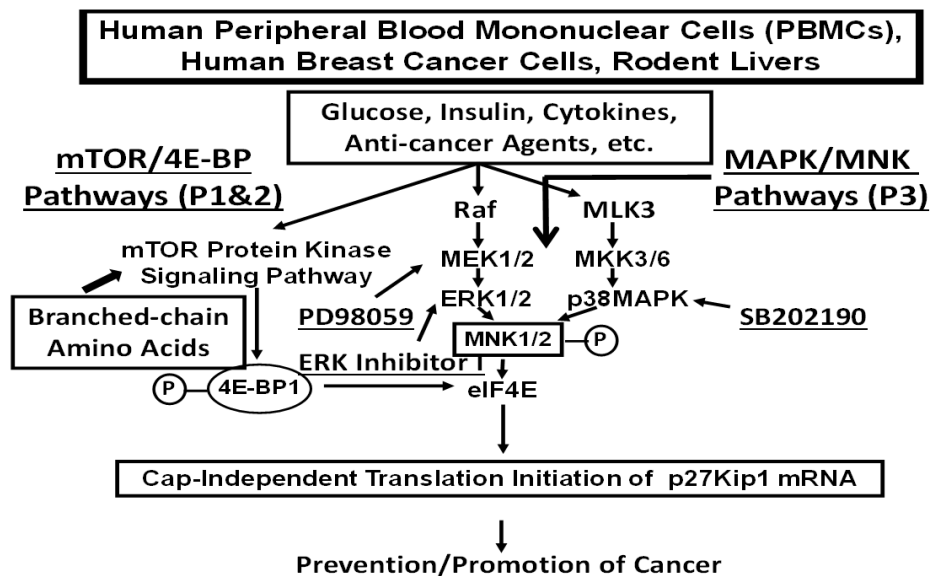

(a)

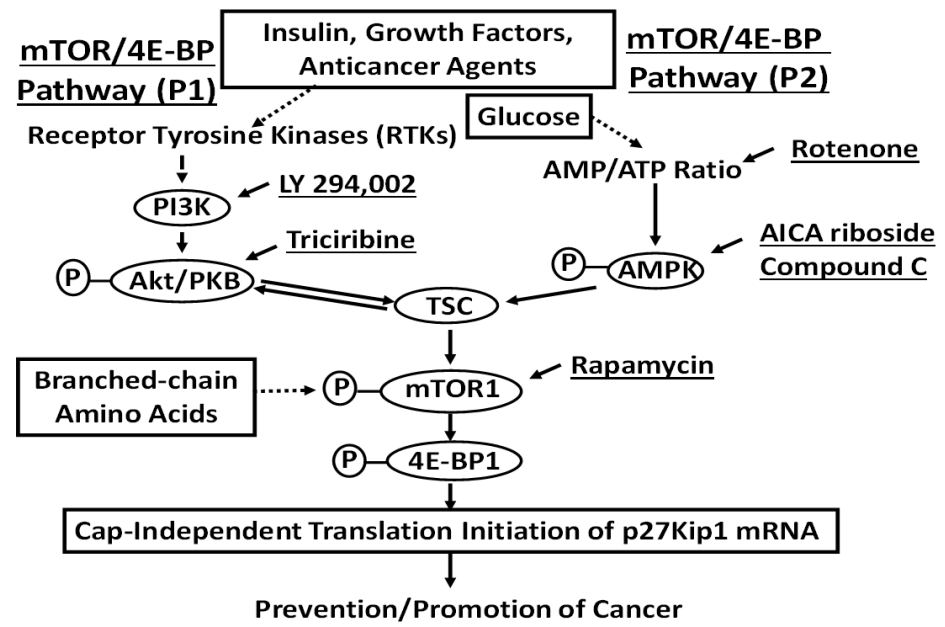

(b)

Figure 4. Regarding the results presented in Figure 3, panels a and b present the hypothetical upstream molecular signaling pathways-including 4E-BP1 and MNK1-of the expression of p27 in human PBMCs obtained from lean normal donors as compared to those in (1) the livers from genetically homozygous obese diabetic rats or mice [2] and (2) human breast cancer cell lines in vitro [12]-[14].

press decreased - by about 40\%-relative to the unfractionated PBMCs. Surprisingly, this extent of the decrease in the expression of MNK1 in the T (CD3+) cells did not interfere with the expression of the p27 itself in the same $\mathrm{T}(\mathrm{CD} 3+)$ cells.

In summary, the results presented above suggested that all sub-populations of PBMCs, including T (CD3+) cells and monocytes (CD14+), probably expressedthe same level of p27 as unfractionated PBMCs in either lean normal African or Caucasian Americans.

\subsection{Severe Depression-By over 95\%-Of the Expression of p27 in the Peripheral Blood Mononuclear Cells (PBMCs) from Obese Type 2 Diabetic African Americans (AAs) Was Caused Primarily by the Severe Depression of MNK1 Upstream Molecular Signaling Pathways of the Expression of p27}

Last but certainly not least, the molecular basis of the cause of the severe depression-by over 95\%—of the expression of p27 in the peripheral blood mononuclear cells (PBMCs) from obese type 2 diabetic African Americans (AAs) relative to the lean normal African Americans (AAs) was investigated next (Panels a to h of Figure 5).

The results obtained (presented in Panels a to h of Figure 5) are summarized in the Panel a of Figure 6 for 


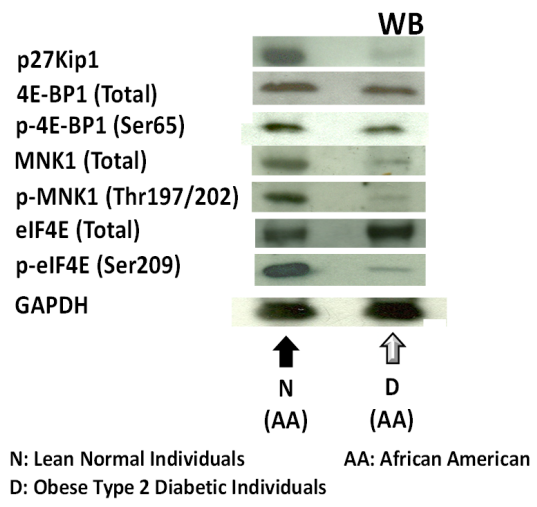

(a)

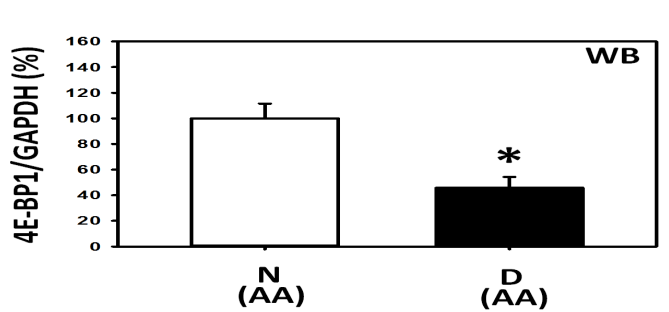

(c)

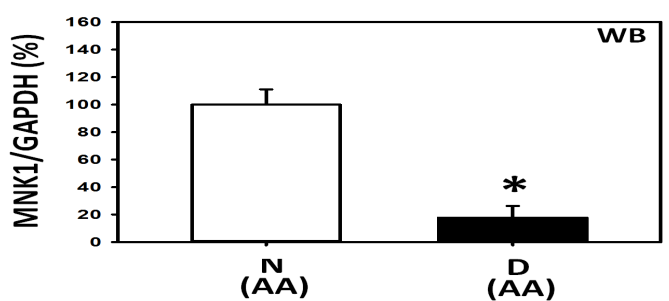

(e)

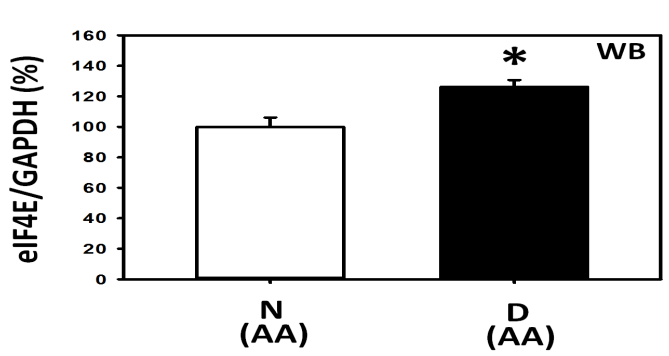

(g)

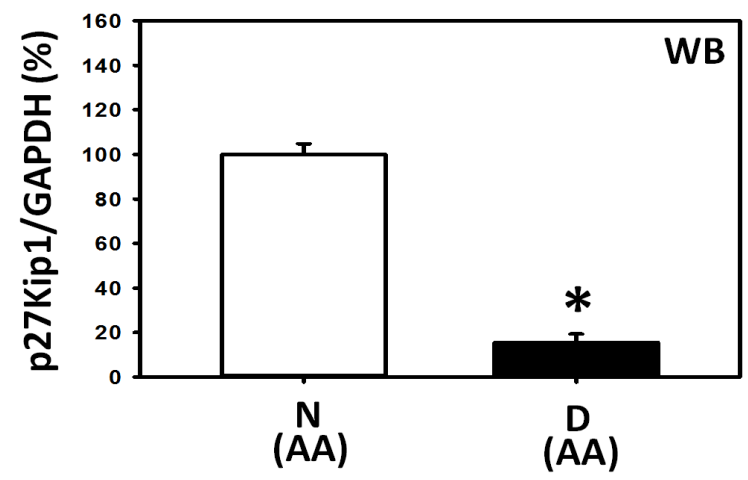

(b)

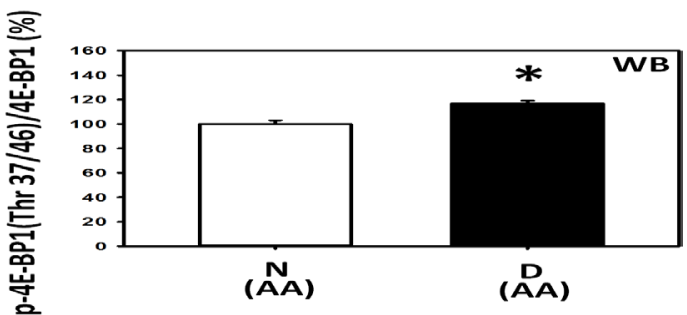

(d)

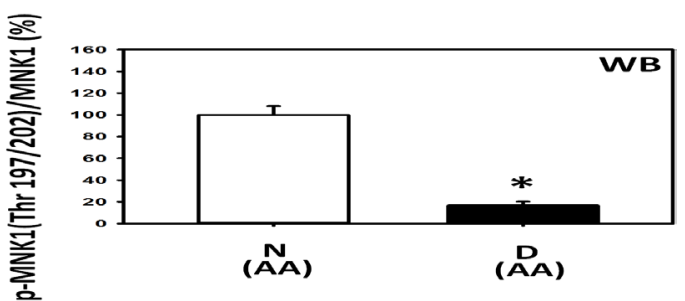

(f)

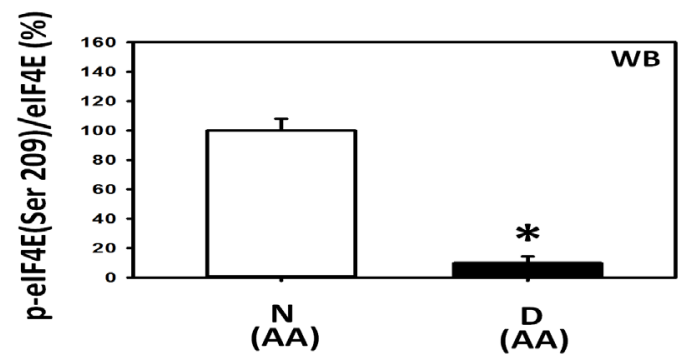

(h)

Figure 5. Severe depression-by over 95\% - of the expression of p27 in the peripheral blood mononuclear cells (PBMCs) obtained from obese type 2 diabetic African Americans (AAs) was probably caused primarily by 1) the severe depression of the MNK1 upstream molecular signaling pathways of the expression of p27 and secondarily by 2) the moderate increase in the phosphorylation of 4E-BP1 upstream molecular signaling pathways of the expression of p27. Panels a to h present the results of the western immunoblot analysis of (Panels a and b) p27 (n = 3), (Panels a and c) total 4E-BP1 $(n=3)$, (Panels a and d) 4E-BP1 phosphorylated at Thr 37/46 ( $=3)$, (Panels a and e) total MNK1 $(n=3)$, (Panels a and f) MNK1 phosphorylated at Thr 197/202 ( $n=3)$, (Panels a and g) total eIF4E $(n=3)$, and (Panels a and h) eIF4E phosphorylated at Ser 209 $(n=3)$.

lean normal African and Caucasian Americans (AAs and CAs) and Panel b of Figure 6 for primarily obese type 2 diabetic African Americans (AAs). Stated briefly, the severe depression of the expression of p27 in the PBMCs from obese type 2 diabetic African Americans was caused primarily by 1) the severe depression of the MNK1 upstream molecular signaling pathways and secondarily by 2) the moderate increase in the phosphoryla- 


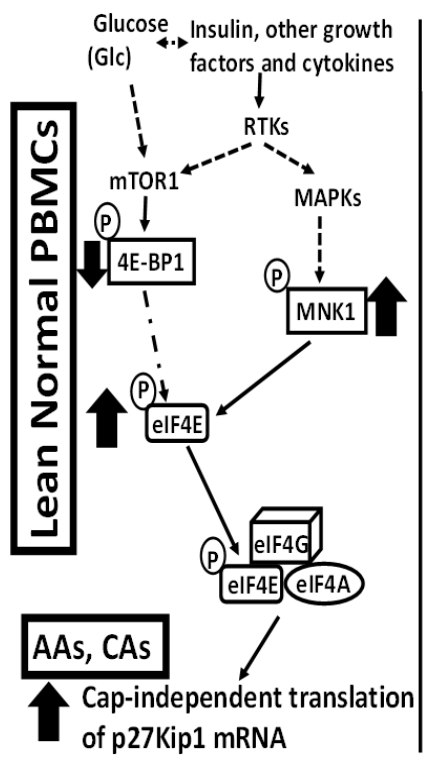

(a)

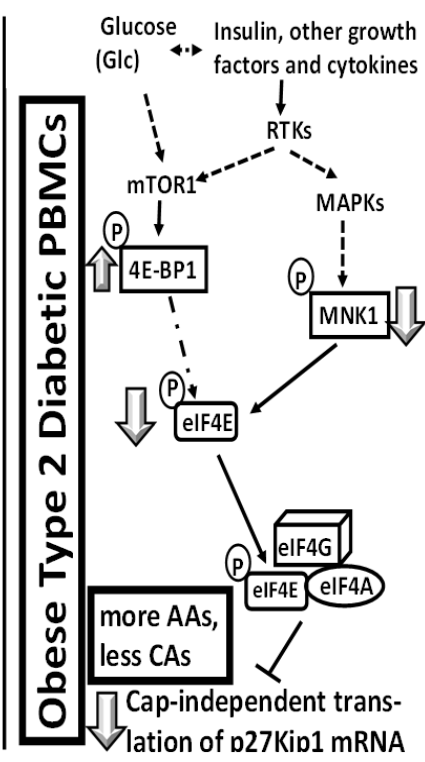

(b)

\begin{abstract}
Figure 6. Regarding the results presented in Figure 5, panels a and b present the hypothetical MNK1 and 4E-BP1 upstream molecular signaling pathways of the expression of p27 in the PBMCs obtained from lean normal African and Caucasian Americans (AAs nd CAs) (Panel (a)) and primarily obese type 2 diabetic African Americans (AAs) (Panel (b)).
\end{abstract}

tion of the 4E-BP1 upstream molecular signaling pathways of the expression of p27.

Panels a, e and f of Figure 5 show that the expressions of total MNK1 and MNK1 phosphorylated at Thr 197/202 in the PBMCs from obese type 2 diabetic African Americans (AAs) were severely depressed-by approximately $82.2 \%$ and $83.3 \%$, respectively-relative to the lean normal African Americans (AAs). The results presented above in the previous section indicated that the depression of MNK1 by approximately $40 \%$ did not significantly decrease the expression of p27. The results presented here in the Panels a and b of Figure 5 suggested that the depression of total MNK1 and MNK1 phosphorylated at Thr 197/202 by over 80\% was associated with the severe depression of the expression of p27-by over 95\% - in the PBMCs from obese type 2 diabetic African Americans (AAs) relative to the lean normal African Americans (AAs). It could have been that the depression of the expression of MNK1 by $40 \%$ was not enough to severely depress the expression of p27.

Lastly, the results presented in the Panels a, $g$ and $\mathrm{h}$ of Figure 5 indicated that the expression of the total eIF4E was significantly increased but the expression of the eIF4E phosphorylated at Ser 209 was severely depressed in the PBMCs from obese type 2 diabetic African Americans (AAs). This observation suggested that the eIF4E unphosphorylated at Ser 209 might protect the 5'-N7-methylguanosine cap (5'- $\mathrm{m}^{7} \mathrm{G}$ cap)in the 5'-untranslated region (5'-UTR) of the p27 mRNA, thereby severely depressing the inherent cap-independent translation initiation of p27 mRNA into p27 protein in obese type 2 diabetic African Americans [13] [15] [16].

\title{
4. Discussion
}

\subsection{Expression of p27(Kip1) Was Significantly Decreased in Human Peripheral Blood Mononuclear Cells (PBMCs) Obtained from Obese Type 2 Diabetic Individuals Relative to the Lean Normal Controls}

The results presented above indicated that the expression of p27 was significantly decreased in human peripheral blood mononuclear cells (PBMCs) from obese type 2 diabetic individuals relative to the lean normal controls. These results were very similar to those obtained with rodent livers, where the expression of p27 in the liver was inversely associated with the potential risk of developing obesity related cancer in obese diabetic rats and mice and the long-lived Ames dwarf mice [2]. Based on the results of this new study, the expression of p27 in human 
PBMCs might be useful as an early biomarker for predicting the potential risk of developing obesity-related cancer in obese type 2 diabetic individuals.

As for the kidney, the levels of the expression of p27 observed in the human PBMCs were the opposite of what was observed in the kidney. One possible way to reconcile this dichotomy might be to regard the expression of p27 in diabetic nephropathy as localized and specialized to the mesangial cells and podocytes in the kidneys. By contrast, the expression of p27 in human PBMCs could be regarded rather as generalized to the whole body due to the circulation of the PBMCs in the blood.

Multiple articles regarding the role of p27 and other factors in the kidney disease have been published [17][29], but one examplemight be cited that suggests the complexity of the underlying molecular mechanisms of the expression of p27 in diabetic nephropathy. In this example, it was proposed that the increased expression of S-phase kinase-associated protein 2 (Skp2) in progressive nephropathy with a consequent Skp2-mediated reduction in p27 might be the pathogenic activity that occurs during the progression of nephropathy [30]. This study was performed in the context that the tubular epithelial cell proliferation had been known to be a characteristic feature of the renal damage that is apparent in the early stages of nephropathy [30]. Similar to the kidney, Skp2-mediated acceleration of p27 degradation was also implicated in the regulation of the proliferation of $\beta$-cells in the pancreas [31]-[33].

\subsection{Expression of p27 in Human PBMCs Appeared to Be Most Severely Depressed-By over 95\%-In Obese Type 2 Diabetic African Americans Relative to the Lean Normal African Americans. By Contrast, the Extent of the Decrease in the Expression of p27 in Human PBMCs Was Quite Variable-By 20\% to 86\%-In Obese Type 2 Diabetic Caucasian Americans Relative to the Lean Normal Caucasian Americans.}

It should be noted here that the levels of the expression of p27 in the PBMCs obtained from lean normal African American controls were not significantly different from those obtained from lean normal Caucasian American controls. This suggested that the observation that the expression of p27 in the PBMCs from obese type 2 diabetic African Americans was so severely depressed is likely to be correct. To investigate the molecular mechanisms of how the expression of p27 in PBMCs was so severely depressed in obese type 2 diabetic African Americans, attention was focused on the activities of the two most relevant upstream molecular signaling pathways of the expression of p27, namely the MNK1 and 4E-BP1 pathways.

The results of this investigation suggested that the severe depression of the expression of p27 in the PBMCs from obese type 2 diabetic African Americans was probably caused primarily by 1) the severe depression of the MNK1 upstream molecular signaling pathways of the expression of p27 and secondarily by 2) the moderate increase in the phosphorylation of the 4E-BP1 upstream molecular signaling pathways of the expression of p27.

The moderate decrease in the expression of p27 by about $20 \%$ was already observed in the rodent livers from obese diabetic rats and mice and found to be caused primarily by the moderate increase in the phosphorylation of the 4E-BP1 upstream molecular signaling pathways of the expression of p27 [2]. Similarly, moderate decrease in the expression of p27 and the moderate increase in the phosphorylation of 4E-BP1 pathways was also observed in the human breast cancer cell lines in vitro where the concentration of glucose, insulin, or branchedchain amino acids was increased moderately in the culture medium [14]. As for the MNK1 pathways, any articles could be found in the literature that reported the severe depression-by over 95\%—of the activities of the MNK1 pathways in obese type 2 diabetes.

It might be speculated that the severe depression of p27 in the PBMCs from obese type 2 diabetic African Americans could have resulted from the following two sequential hits, namely the first hit on the 4E-BP1 phosphorylation pathways, and the second hit on the MNK1 pathways. We already know that the first hit was originated from the increased concentration of glucose, insulin or branched-chain amino acids [2] [14], but it is not yet known where the second hit was originated from.

\subsection{Diabetes-Cancer Link}

The American Institute for Cancer Research released a statement about the diabetes-cancer link. It stated that the “estimated 100 million Americans at risk for or with diabetes are also at increased risk of several types of cancer”. Furthermore, the American Diabetes Association and the American Cancer Society issued a consensus report on diabetes and cancer [1]. It stated that in "the US, African Americans are more likely to develop and die 
from cancer than other race or ethnic groups" and further that in "the US, type 2 diabetes and its complications disproportionately affect a number of specific populations including African Americans compared with nonHispanic whites" and while "incompletely understood, genetic, socioeconomic, lifestyle, and other environmental factors are thought to contribute to these disparities".

\section{Acknowledgements}

The author greatly appreciates the research environment provided by the Nutrition Obesity Research Center (P30 DK056336) and the Diabetes Research and Training Center (P60 DK079626), both at the University of Alabama at Birmingham (UAB) (Birmingham, AL, USA). The content of this study, however, is solely the responsibility of the author and does not necessarily represent the official views of the National Institutes of Health or the National Institute of Diabetes and Digestive and Kidney Diseases.

\section{Conflicts of Interest}

The author declares that no actual or potential conflicts of interest exist that might, in principle, influence the author's scientific judgment.

\section{Each Author's Contribution}

Single author contributed the entire work described in this manuscript.

\section{References}

[1] Giovannucci, E., Harlan, D.M., Archer, M.C., Bergenstal, R.M., Gapstur, S.M., Habel, L.A., Pollak, M., Regensteiner, J.G. and Yee, D. (2010) Diabetes and Cancer: A Consensus Report. Diabetes Care, 33, 1674-1685. http://dx.doi.org/10.2337/dc10-0666

[2] Eto, I. (2013) Expression of p27Kip1, A Cell Cycle Repressor Protein, Is Inversely Associated with Potential Carcinogenic Risk in the Genetic Rodent Models of Obesity and Long-Lived Ames Dwarf Mice. Metabolism, 62, 873-888. http://dx.doi.org/10.1016/j.metabol.2013.01.001

[3] Hakkak, R., Holley, A.W., Bunn, R.C., Winters, A. and MacLeod, S. (2005) Effects of Obesity on Serum Insulin Growth Factor 1 (IGF-1) Levels in Lean and Obese Female Zucker Rats Following DMBA Treatment. The FASEB Journal-Proceedings, 19, A993.

[4] Hakkak, R., Holley, A.W., Gnoand, F. and Owens, R. (2005) Effects of Obesity on Serum Adiponectin Levels and Breast Cancer Development in Lean and Obese Female Zucker Rats Following DMBA Ttreatment. The FASEB Journal-Proceedings, 19, A993.

[5] Hakkak, R., Holley, A.W., MacLeod, S., Simpson, P. and Korourian, P. (2005) Obesity Promotes DMBA Induced Mammary Tumor Development in Female Zucker Rats. The FASEB Journal-Proceedings, 19, A774.

[6] Hakkak, R., Holley, A.W., MacLeod, S., Simpson, P., Fuchs, G., Jo, C.H., Kieber-Emmons, T. and Korourian, S. (2005) Obesity Promotes7,12-Dimethylbenz(a)anthracene-Induced Mammary Tumor Development in Female Zucker Rats. Breast Cancer Research, 7, R627-R633. http://dx.doi.org/10.1186/bcr1263

[7] Hakkak, R., Shaaf, S., Jo, C.H., MacLeod, S. and Korourian, S. (2010) Dehydroepiandrosterone Intake Protects against 7,12-Dimethylbenz(a)anthracene-Induced Mammary Tumor Development in the Obese Zucker Rat Model. Oncology Report, 24, 357-362. http://dx.doi.org/10.3892/or_00000867

[8] Hakkak R., MacLeod, S., Shaaf, S., Holley, A. W., Simpson, P., Fuchs, G., Jo, C.H., Kieber-Emmons, T. and Korourian, S. (2007) Obesity Increases the Incidence of 7,12-Dimethylbenz(a)anthracene-Induced Mammary Tumors in Ovariectomized Zucker Rat. International Journal of Oncology, 30, 557-563.

[9] Whitehead, T., Holley, A.W., Kieber-Emmons, T., Korourian, S. and Hakkak, R. (2005) Metabolic Phenotype of the DMBA-induced Mammary Tumor in Obese Female Zucker Rats. Proceedings of the American Association for Cancer Research, 46, 6074.

[10] Ikeno, Y., Bronson, R.T., Hubbard, G.B., Lee, S. and Bartke, A. (2003) Delayed Occurrence of Fatal Neoplastic Diseases in Ames Dwarf Mice: Correlation to Extended Longevity. Journal of Gerontology Series A, 58, B291-B296. http://dx.doi.org/10.1093/gerona/58.4.B291

[11] Sharp, Z.D. and Bartke, A. (2005) Evidence for Down-Regulation of Phosphoinositide 3-Kinase/Akt/Mammalian Target of Rapamycin (PI3K/Akt/mTOR)-Dependent Translation Regulatory Signaling Pathways in Ames Dwarf Mice. Journal of Gerontology Series A, 60, 293-300.

[12] Eto, I. (2006) Nutritional and Chemopreventive Anti-Cancer Agents Up-Regulate Expression of p27Kip1, A Cy- 
clin-Dependent Kinase Inhibitor, in Mouse JB6 Epidermal and Human MCF7, MDAMB-321 and AU565 Breast Cancer Cells. Cancer Cell International, 6, 1-19. http://www.cancerci.com/content/6/1/20 http://dx.doi.org/10.1186/1475-2867-6-20

[13] Eto, I. (2010) Upstream Molecular Signaling Pathways of p27(Kip1) Expression: Effects of 4-Hydroxytamoxifen, Dexamethasone, and Retinoic Acids. Cancer Cell International, 10, 1-19. http://www.cancerci.com/content/10/1/3 http://dx.doi.org/10.1186/1475-2867-10-3

[14] Eto, I. (2011) Upstream Molecular Signaling Pathways of p27(Kip1) Expression in Human Breast Cancer Cells in Vitro: Differential Effects of 4-Hydroxytamoxifen and Deficiency of Either D-(+)-Glucose or L-Leucine. Cancer Cell International, 11, 1-17. http://www.cancerci.com/content/11/1/31 http://dx.doi.org/10.1186/1475-2867-11-31

[15] Kullmann, M., Goepfert, U., Siewe, B. and Hengst, L. (2002) ELAV/Hu Proteins Inhibit p27 Translation via an IRES Element in the p27 5'UTR. Genes \& Development, 16, 3087-3099. http://dx.doi.org/10.1101/gad.248902

[16] Goepfert, U., Kullmann, M. and Hengst, L. (2003) Cell Cycle-Dependent Translation of p27 Involves a Responsive Element in Its 5'-UTR That Overlaps with a uORF. Human Molecular Genetics, 12, 1767-1779. http://dx.doi.org/10.1093/hmg/ddg177

[17] Awazu, M., Omori, S., Ishikura, K., Hida, M. and Fujita, H. (2003) The Lack of CyclinKinase Inhibitor p27 ${ }^{\text {Kip1 }}$ Ameliorates Progression of Diabetic Nephropathy. Journal of the American Society of Nephrology, 14, 699-708. http://dx.doi.org/10.1097/01.ASN.0000051726.41601.C0

[18] Wolf, G. and Shankland, S.J. (2003) p27 Kip1: The "Rosebud" of Diabetic Nephropathy? Journal of the American Society of Nephrology, 14, 819-922. http://dx.doi.org/10.1097/01.ASN.0000057518.58420.E4

[19] Wolf, G., Schroeder, R., Thaiss, F., Ziyadeh, F.N., Helmchen, U. and Stahl, R.A. (1998) Glomerular Expression of p27 ${ }^{\text {Kip } 1}$ in Diabetic $d b / d b$ Mouse: Role of Hyperglycemia. Kidney International, 53, 869-879. http://dx.doi.org/10.1111/j.1523-1755.1998.00829.x

[20] Shankland, S.J. (1998) The Growing Role for the Cyclin Kinase Inhibitor p $27^{\mathrm{Kip} 1}$ in Renal Disease. Kidney International, 54, 2241-2242. http://dx.doi.org/10.1038/4499991

[21] Wolf, G. (2000) Cell Cycle Regulation in Diabetic Nephropathy. Kidney International, 58, S59-S66. http://dx.doi.org/10.1046/j.1523-1755.2000.07710.x

[22] Wolf, G., Schanze, A., Stahl, R.A., Shankland, S.J. and Amann, K. (2005) p27 ${ }^{\text {Kip } 1}$ Knockout Mice Are Protected from Diabetic Nephropathy: Evidence for p27 ${ }^{\text {Kip1 }}$ Haplotype Insufficiency. Kidney International, 68, 1583-1589. http://dx.doi.org/10.1111/j.1523-1755.2005.00570.x

[23] Wolf, G. and Ziyadeh, F.N. (2007) Cellular and Molecular Mechanisms of Proteinuria in Diabetic Nephropathy. Nephron Physiololgy, 106, 26-31. http://dx.doi.org/10.1159/000101797

[24] Rüster, C. and Wolf, G. (2006) Renin-Angiotensin-Aldosterone System and Progression of Renal Disease. Journal of the American Society of Nephrology, 17, 2985-2991. http://dx.doi.org/10.1681/ASN.2006040356

[25] Wolf, G. (2006) Renal Injury Due to Renin-Angiotensin-Aldosterone System Activation of the Transforming Growth Factor- $\beta$ Pathway. Kidney International, 70, 1914-1919.

[26] Loeffle, I., Hopfer, U., Koczan, D. and Wolfe, G. (2011) Type VIII Collagen Modulates TGF- $\beta 1$-Induced Proliferation of Mesangial Cells. Journal of the American Society of Nephrology, 22, 649-663. http://dx.doi.org/10.1681/ASN.2010010098

[27] Loeffler, I., Rüster, C., Franke, S., Liebisch, M. and Wolf, G. (2013) Erythropoietin Ameliorates Podocyte Injury in Advanced Diabetic Nephropathy in the $d b / d b$ Mouse. American Journal of Physiology-Renal Physiology, 305, F911F918. http://dx.doi.org/10.1152/ajprenal.00643.2012

[28] Kanwar, Y.S., Wada, J., Sun, L., Xie, P., Wallner, E.I., Chen, S., Chugh, S. and Danesh, F.R. (2008) Diabetic Nephropathy: Mechanisms of Renal Disease Progression. Experimental Biology and Medicine (Maywood), 233, 4-11. http://dx.doi.org/10.3181/0705-MR-134

[29] Wolf, G. (Ed.) (2006) Obesity and the Kidney. Basel, Karger.

[30] Suzuki, S., Ohashi, N. and Kitagawa, M. (2013) Roles of the Skp2/p27 Axis in the Progression of Chronic Nephropathy. Cellular and Molecular Life Sciences, 70, 3277-3287. http://dx.doi.org/10.1007/s00018-012-1232-X

[31] Zhong, L., Georgia, S., Tschen, S.I., Nakayama, K. and Bhushan, A. (2007) Essential Role of Skp2-Mediated p27 Degradation in Growth and Adaptive Expansion of Pancreatic $\beta$ Cells. Journal of Clinical Investigation, 117, 28692876. http://dx.doi.org/10.1172/JCI32198

[32] Tschen, S.I., Georgia, S., Dhawan, S. and Bhushan, A. (2011) Skp2 Is Required for Incretin Hormone-Mediated $\beta$-Cell Proliferation. Molecular Endocrinology, 25, 2134-2143. http://dx.doi.org/10.1210/me.2011-1119

[33] Georgia, S. and Bhushan, A. (2006) p27 Regulates the Transition of $\beta$-Cells from Quiescence to Proliferation. Diabetes, 55, 2950-2956. http://dx.doi.org/10.2337/db06-0249 


\section{Abbreviations}

PBMC, peripheral blood mononuclear cells;

HIV, human immunodeficiency virus;

HTLV, human T-lymphotropic virus;

CMV, cytomegalovirus;

FDA, Food and Drug Administration;

AA, African American;

CA, Caucasian American;

BMI, body mass index;

$\mathrm{CD}$, cluster of differentiation (cluster of designation) antigen;

Hgb A1c, glycated hemoglobin;

DMEM, Dulbecco's Modified Eagle's Medium;

RIPALysis Buffer, radioimmunoprecipitation assaylysis buffer;

PMSF, phenylmethylsulfonyl fluoride;

p27Kip1, cyclin-dependent kinase inhibitor 1B;

p27, p27Kip1;

4E-BP1, eukaryotic translation initiation factor 4E-binding protein 1;

MNK1, MAP kinase-interacting serine/threonine-protein kinase 1;

eIF4E, eukaryotic translation initiation factor $4 \mathrm{E}$;

GAPDH, glyceraldehyde 3-phosphate dehydrogenase;

Thr, threonine;

Ser, serine;

SDS, sodium dodecyl sulfate or sodium lauryl sulfate;

PAGE, polyacrylamide gel electrophoresis;

AP, alkaline phosphatase;

5'-m ${ }^{7} \mathrm{G}$ cap, 5'-N7-methylguanosine cap;

5'-UTR, 5'-untranslated region;

DMBA, 7,12-dimethylbenz(a)anthracene 
Scientific Research Publishing (SCIRP) is one of the largest Open Access journal publishers. It is currently publishing more than 200 open access, online, peer-reviewed journals covering a wide range of academic disciplines. SCIRP serves the worldwide academic communities and contributes to the progress and application of science with its publication.

Other selected journals from SCIRP are listed as below. Submit your manuscript to us via either submit@scirp.org or Online Submission Portal.
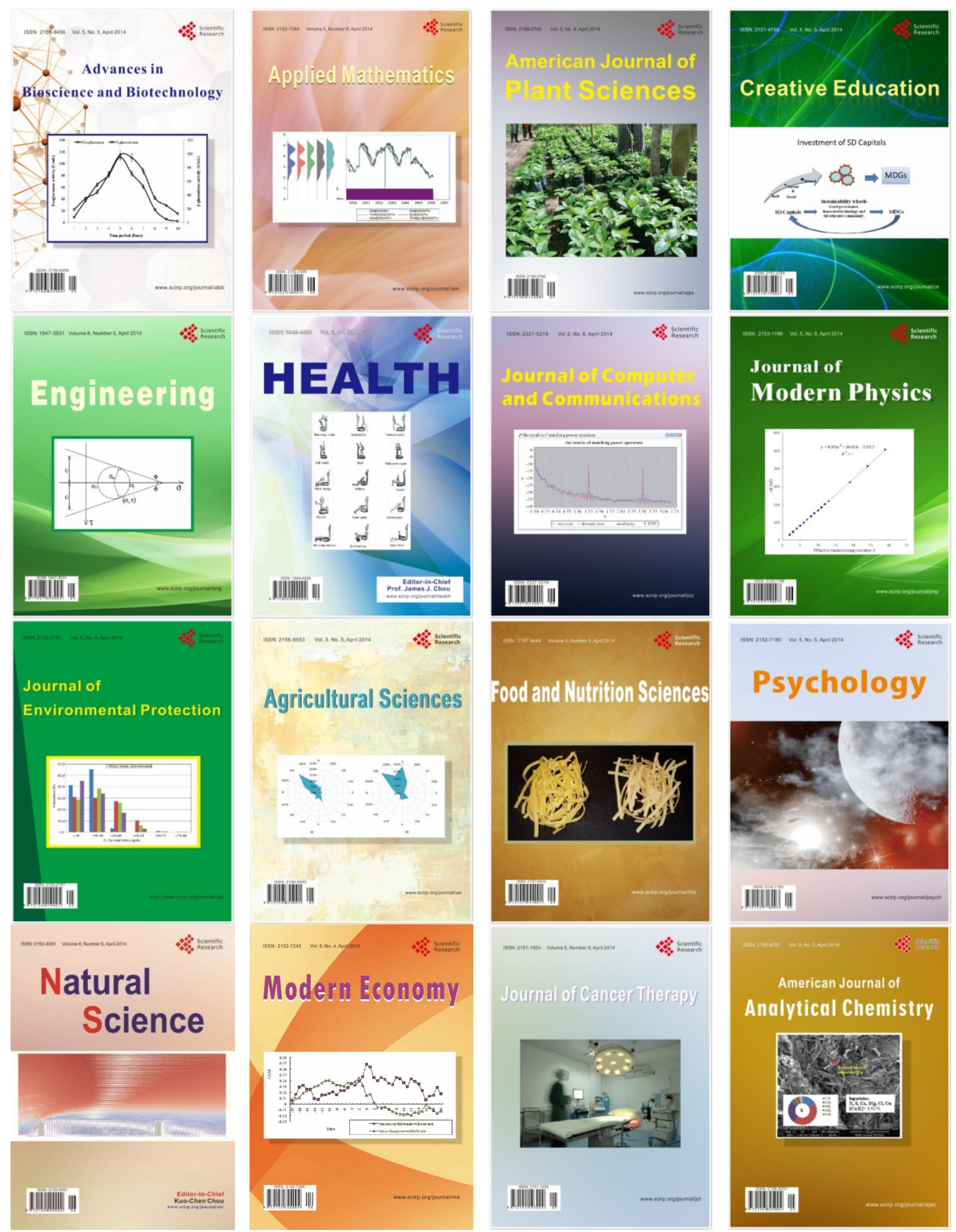